\title{
Hábitos de estudio y ansiedad en estudiantes andragogos
}

\section{Study Habits and Anxiety in Andragogic Students}

\author{
Rosa Esther Montes-Valer \\ Universidad Nacional Mayor de San Marcos, Lima, Perú \\ ORCID: https://orcid.org/0000-0002-8288-0413
}

Recibido 10-02-20 Revisado 12-04-20 Aprobado 23-06-20 En línea 01-07-20

*Correspondencia

Email: roemontes@hotmail.com

\section{Citar como:}

Montes-Valer, R. (2020). Hábitos de estudio y ansiedad en estudiantes andragogos. Propósitos y Representaciones, 8(2), e560. doi: http://dx.doi.org/10.20511/pyr2020.v8n2.560 


\section{Resumen}

El objetivo de la presente investigación fue determinar la relación entre hábitos de estudio y autoevaluación de la ansiedad en estudiantes andragogos. El diseño de la investigación fue no experimental transversal de tipo comparativo. La población de estudio estuvo conformada por 180 estudiantes de la carrera de Administración del programa "Working Adult" de la Universidad Privada del Norte (UPN), matriculados en el primer ciclo del curso Habilidades Comunicativas y el tamaño de la muestra fue de 139 estudiantes. Para la medición de las variables de investigación, se utilizó la autoevaluación de la ansiedad (elaborado por Zung), el inventario de Hábitos de estudio (elaborado por Luis Vicuña Peri). Se encontró una correlación negativa significativa entre ambas variables, lo que significa que el grupo de personas que reportaron mayores niveles de ansiedad presentaron a su vez menores índices de hábitos de estudio.

Palabras clave: Aprendizaje de adultos; Organización y método de estudio; Ansiedad; Educación superior.

\section{Summary}

The objective of this research was to determine the relationship between study habits and selfassessment of anxiety in andragogic students. The research design was non-experimental, crosssectional of comparative type. The study population consisted of 180 students of the Administration career of the "Working Adult" program of the Private University of the North (UPN), enrolled in the first cycle of the Communication Skills course and the sample size was 139 students. For the measurement of the research variables, the anxiety self-assessment (prepared by Zung), the study Habits inventory (prepared by Luis Vicuña Peri) was used. A significant negative correlation was found between both variables, which means that the group of people who reported higher levels of anxiety had lower rates of study habits.

Keywords: Adult Learning; Organization and Study Method; Anxiety; Higher Education

\section{Introducción}

La andragogía es considerada la ciencia y arte de la educación de adultos ya que engloba las características del adulto en situación de aprendizaje, proporcionando el carácter científico a la educación de adultos (Alcalá, 1999). La Andragogía es parte de la Antropología y se desarrolla a través de ciertas variables: institución educativa, ambiente, el contrato de aprendizaje, la didáctica y la evaluación y el trabajo en quipos cuyo proceso permite incrementar el pensamiento, la autogestión, la calidad de vida y la creatividad en el adulto con el fin de brindarle la oportunidad para que logre su autorrealización (Chilca, 2017).

La UNESCO (1976) menciona que la educación de adultos es una actividad compleja y que según sus modalidades y lugares donde se ejerce, toma diversas denominaciones y se presenta de distintas maneras, existiendo al mismo tiempo el interés por conceptualizar dando lugar a la aparición de una serie de definiciones. Podemos mencionar: educación continua, educación popular, Andragogía, educación informal, extensión educativa, desarrollo comunitario, educación profesional permanente, educación permanente, alfabetización, educación de la tercera edad, educación de jóvenes y adultos y últimamente en nuestro país educación básica alternativa, etc (Tahua \& Cecilia, 2018).

En el Perú, se ha realizado propuestas direccionadas a la andragogía observándose que los programas existentes dificultan de alguna manera la aplicación práctica y significativa de lo aprendido por parte de los estudiantes. Eso se torna lamentable teniendo en ese espacio una posibilidad muy valiosa para formular y enriquecer las prácticas cotidianas, es necesario, entonces, establecer qué rumbo queremos tomar y a dónde queremos dirigirnos (Sandoval, 2019; 
Alarcón-Diaz, Zapata, Díaz, Fernández, Hernández, \& Rodríguez. 2018). Lo mencionado anteriormente nos muestra la importancia de la elaboración de un Modelo Curricular Andragógico que ayudará a operacionalizar a la Andragogía como ciencia y las hipótesis y principios en los que se sustenta (Alcalá, 1999; Silva, 2018).

Un hábito, se adquiere por la repetición, convirtiéndose en parte de la personalidad y bajo este proceso es que se hace una costumbre y se desarrolla en dos etapas donde en la primera se adquiere el hábito y en la segunda se afianza, es decir, se vuelve automático y se realiza de manera habitual (Paredes, 2018).

Según Cruz y Quiñones, (2011), los hábitos de estudio son costumbres y formas de reconocer, captar, juzgar y pensar. El hecho que te hace el ser estudiante implica tener metas de estudio bien establecidos, pero sobretodo actitud frente al conocimiento sumado el tener un adecuado manejo del tiempo, la planificación del estudio y conocer y practicar métodos y técnicas que le permitirán al estudiante lograr el tan ansiado éxito académico (Hernández, Rodríguez,\& Vargas, 2012; Suárez Vera, 2018).

Mientras la ansiedad, según la definición del Diagnostic and Statistical Manual of Mental Disorders (DSM -V), es una anticipación aprensiva de un peligro o desgracia futura acompañada de un sentimiento de malestar o síntomas somáticos de tensión. Para Salazar García, (2018) la ansiedad es una defensa creada frente a estímulos que rompen el equilibrio fisiológico y psicológico. Es un estado natural y en ocasiones resulta beneficioso o incluso una respuesta saludable (Agudelo, Casadiegos \& Sánchez, 2008; Salazar García, 2018).

Cruz y Quiñones (2011) que realizaron una investigación para determinar la relación que existe entre los hábitos de estudio y el rendimiento académico en estudiantes universitarios de México a fin de brindar alternativas que ayuden al incremento del rendimiento académico y personal del estudiante. El resultado de esta investigación arrojó que el 85\% de estudiantes poseían hábitos de estudio regulares y el $15 \%$ poseían hábitos deficientes. Con respecto al rendimiento académico se halló que el $23 \%$ es excelente, el $62 \%$ es de bueno a regular y solo el $15 \%$ tiene rendimiento académico bajo y al correlacionar ambas variables se observó correlación significativa entre ellos. Se concluye que es imprescindible diseñar una estrategia metodológica para trabajar los hábitos de estudio desde que el estudiante ingresa a la universidad para que obtenga un rendimiento académico adecuado y pueda hacer frente a las exigencias que implican el trabajo académico en los claustros universitarios.

Chilca (2017) realizó una investigación para determinar la relación entre la autoestima, hábitos de estudio con el rendimiento en estudiantes universitarios en Perú. Los resultados concluyeron que no existe una influencia significativa entre la autoestima y el rendimiento académico, pero sí entre los hábitos de estudio y el rendimiento académico de los estudiantes donde se concluye que si los estudiantes mejoraran sus técnicas de estudio se incrementaría su rendimiento académico universitario.

Por lo descrito se pone en manifiesto que los problemas en la andragogía se tornarían mucho más difíciles y complicados para el estudiante andragogo que por sus propias características son personas cuyas edades oscilan entre los 30 a 55 años y que en su momento abandonaron la vida universitaria o nunca asistieron a clases en una escuela de educación superior o solo accedieron a una educación técnica, sin embargo, sus experiencias de vida y conocimientos técnicos justamente por esas experiencias les brindan el manejo adecuado en las diferentes empresas o instituciones en las que laboran, no significando que en los estudios obtengan esa misma performance ni menos que su rendimiento académico sea óptimo.

Los estudiantes andragogos tienen un proceso de aprendizaje y retención de la información diferentes a los que encontramos en jóvenes universitarios, la experiencia que cada uno de los estudiantes andragogos tienen acerca del campo profesional en el cual se desenvuelven 
marca un historial muy importante acerca de sus conocimientos y éstos le ayudan a enfrentar una carrera universitaria, sin embargo, la manera del procesamiento en cuanto a sus procesos cognitivos se ve mermada por la falta de continuidad en el estudio y por su edad. Por tal motivo es importante conocer de cerca este fenómeno, donde los estudiantes andragogos tienen por un lado la motivación de iniciar estudios universitarios y, por otro lado, las dificultades de enfrentarlos de manera satisfactoria y que lamentablemente contribuyen al establecimiento de estados ansiógenos, estrés, etc y que conlleva al abandono de los estudios. La presente investigación busca determinar la relación que existe entre los hábitos de estudio y la ansiedad en los estudiantes andragogos en una universidad peruana.

\section{Método}

La investigación corresponde a un diseño no experimental transversal de tipo comparativo que pretende encontrar la relación entre Hábitos de estudio y autoevaluación de la ansiedad en estudiantes andragogos de una universidad de Lima.

Los sujetos participantes fueron 139 estudiantes andragogos (70 varones y 69 mujeres) de la carrera de Administración y Negocios Internacionales.

Para la delimitación de los sujetos de la muestra del estudio se siguió los siguientes criterios de inclusión y exclusión.

\section{Criterios inclusión}

- Estudiantes que han concluido sus estudios secundarios en los CEBA o que no han concluido sus estudios técnicos.

- Alumnos del primer ciclo de la carrera de Administración y Negocios Internacionales

Criterios exclusión

- Estudiantes de pregrado regular

- Estudiantes con segunda carrera

Para la evaluación se aplicó el Inventario de Hábitos de Estudio CASM 85. Revisión 2014, (Vicuña, 2014). El inventario se administra de manera individual y colectiva su objetivo es la medición y diagnóstico de los Hábitos de Estudio de alumnos de Educación Secundaria y de los primeros años de la universidad y/o institutos superiores. Está constituido por 55 ítems distribuido en 5 áreas que a continuación se detalla:

\begin{tabular}{lll}
\hline & \multicolumn{2}{c}{ Inventario de Hábitos de Estudio } \\
\hline Área I & ¿Cómo estudia usted? & Constituida por 12 ítems \\
\hline Área II & ¿Cómo hace sus tareas? & Constituida por 10 ítems \\
\hline Área III & ¿Cómo prepara sus exámenes? & Constituida por 11 ítems \\
\hline Área IV & ¿Cómo escucha sus clases? & Constituida por 13 ítems \\
\hline Área V & ¿Qué acompaña sus momentos de estudio? & Constituida por 9 ítems \\
\hline
\end{tabular}

\section{Instrumentos}

\section{Inventario de Hábitos de Estudio}

El inventario por el método de las mitades (Split Half) presenta un coeficiente de 0.86 para todo el inventario y coeficientes significativos al 0.01 en cada área. La validez se realizó en dos etapas, la primera con el propósito de seleccionar las 120 conductas que los estudiantes comunican poner en acción cuando estudian, sólo aquellas que significativamente polarizan, y la segunda etapa para decidir la dirección de la respuesta que será calificada como adecuada o inadecuada. 
Para la primera etapa, una vez aplicado el inventario, la muestra intencionalmente fue categorizada en base al rendimiento académico en dos grupos: el primero estuvo conformado por los estudiantes con alto rendimiento académico y otro por estudiantes de bajo rendimiento académico; para lo cual se utilizó como criterio de clasificación los cuartiles de modo que los primeros estuvieron comprendidos dentro de cuartil 3 y el segundo grupo de cuartil 1, luego se tabuló las respuestas "SI" para cada ítem, de esa manera obtendríamos las proporciones acordes con el criterio y siempre que la proporción fuese igual o menor de 0.42 o también igual o mayor de 0.58. En estos casos, se retiene el ítem por la siguiente razón: la diferencia de proporciones para 0.42 y 0.58 es igual a 0.16 con un error estándar de 0.08 que arroja una razón critica de diferencia de proporciones igual a 2 que para 78 grados de libertad al 0.05 el valor teórico para pruebas de dos extremos es igual a 1.99 por tanto el margen de diferencia es significativo. Con este criterio los 120 complejos conductuales quedaron eliminados 67 ítems, debiendo retenerse sólo 55 .

En la segunda etapa, con estos 55 ítems pasamos a darle la dirección de adecuado o positivo y de inadecuado o negativo, para tal fin no hemos utilizado el criterio de expertos, se ha preferido utilizar el criterio de discriminación del ítem para lo cual la designación de la respuesta como adecuada o inadecuada debe partir del dominio de elección de los examinados, así, una respuesta a un ítem será considerada como positiva así corresponde significativamente al grupo de alto rendimiento académico.

\section{Autoevaluación de la Escala de Ansiedad de Zung}

El estudio realizado en el año 1971 con 225 pacientes psiquiátricos y 343 no pacientes, obtuvo 0.66 para no pacientes y 0.74 para pacientes diagnosticados con desordenes de ansiedad dicho estudio ayudó para la obtención de la validez de esta escala. La confiabilidad de esta escala fue obtenida mediante el método de dos mitades.71, y alfa fue de .85. En el Perú Astocondor lo validó en el año 2011. Para ello se utilizó el método de validez interna por criterio de jueces, usando el Coeficiente V de Aiken arrojando valores entre 0.8 y 1 para todos los ítems con significancia de .05, para ello se utilizó Alpha de Cronbach obteniendo un puntaje de .78.

\section{Procedimiento}

El investigador a cargo administró los instrumentos de manera colectiva. Se tuvo sumo cuidado con el manejo de la información, siguiendo de esta manera, los protocolos de los principios éticos. Es así, que la participación fue voluntaria y anónima, previa a la administración de los instrumentos se entregó a cada participante un consentimiento informado, para su respectiva lectura y firma. Se dieron a conocer los objetivos de la investigación informando que los datos serian analizados sin identificar al participante y que su tratamiento sería confidencial. La evaluación tuvo lugar en las instalaciones de la universidad en una fecha y horario acordados previamente entre el investigador, los participantes y la dirección del programa académico tomado en cuenta la infraestructura y mobiliario básico (escritorio, sillas, iluminación, privacidad, etc). Para la elaboración de resultados se utilizó el software SPSS v.22.

\section{Resultados}

Se inició el análisis con los resultados de los hábitos de estudio. El inventario de hábitos de estudio está constituido por cinco áreas: ¿Cómo estudia Usted?,¿Cómo hace sus tareas? ¿Cómo prepara sus exámenes? ¿Cómo escucha las clases? ¿Qué acompaña sus momentos de estudio? Encontrando que los datos no se distribuyen conforme a la normal estadística, utilizándose para contrastar los datos la prueba U de Mann Whitney cuya comparación de rangos produjo valores $\mathrm{Z}$ cuya significación asintótica al 0,05 de margen de error, no han resultado menores de 0.05 , por lo que se afirma que esta variable no difiere significativamente del grupo de varones y mujeres, los resultados dejen entrever, que el promedio de hábitos positivos en general corresponde a la 
categoría de estudiantes con pocos hábitos positivos de estudio, por lo que se presupone que tendrán inconvenientes para enfrentar con éxito la tarea universitaria (Tabla1).

Tabla 1.

Descriptivo de valores de Hábitos de estudio.

\begin{tabular}{|c|c|c|c|c|c|c|c|c|c|}
\hline Grupo & & $\mathrm{N}$ & $\begin{array}{l}\text { Rango } \\
\text { promedio }\end{array}$ & $\begin{array}{l}\text { Suma } \\
\text { de } \\
\text { rangos }\end{array}$ & Media & $\begin{array}{l}\text { Desviación } \\
\text { Estandar }\end{array}$ & $\begin{array}{l}\text { U de } \\
\text { Mann- } \\
\text { Whitney }\end{array}$ & Z & $\begin{array}{l}\text { Sig. } \\
\text { asintótica } \\
\text { (bilateral) }\end{array}$ \\
\hline \multirow{2}{*}{$\begin{array}{l}\text { I. ¿Cómo } \\
\text { estudia Usted? }\end{array}$} & & 79 & 37,39 & 1346,00 & 6,7778 & 2,13957 & 680,000 & $-0,044$ & 0,965 \\
\hline & Mujeres & 60 & 37,61 & 1429,00 & 6,7632 & 2,46526 & & & \\
\hline
\end{tabular}

\begin{tabular}{|c|c|c|c|c|c|c|c|c|c|}
\hline \multirow{2}{*}{$\begin{array}{l}\text { II. ¿Cómo } \\
\text { haces sus } \\
\text { tareas? }\end{array}$} & Hombres & 79 & 42,49 & 1529,50 & 8,0000 & 1,62129 & 504,500 & $-1,975$ & 0,058 \\
\hline & Mujeres & 60 & 32,78 & 1245,50 & 7,1316 & 1,90548 & & & \\
\hline \multirow{2}{*}{$\begin{array}{l}\text { III. ¿Cómo } \\
\text { prepara sus }\end{array}$} & Hombres & 79 & 38,97 & 1403,00 & 7,5000 & 2,00713 & 631,000 & $-0,579$ & 0,562 \\
\hline & Mujeres & 60 & 36,11 & 1372,00 & 7,1842 & 1,98447 & & & \\
\hline
\end{tabular}

exámenes?

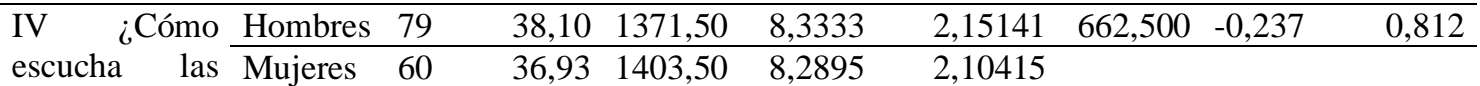

clases?

\begin{tabular}{llllllllll}
\hline V. ¿Qué & Hombres & 79 & 39,72 & 1430,00 & 6,3056 & 1,67023 & 604,000 & $-0,887$ & 0,375 \\
\cline { 2 - 10 } $\begin{array}{l}\text { acompaña sus } \\
\text { momentos de } \\
\text { estudio? }\end{array}$ & Mujeres & 60 & 35,39 & 1345,00 & 6,0000 & 1,75530 & & & \\
\hline Total General & Hombres & 79 & 40,10 & 1443,50 & 36,9167 & 7,05641 & 590,500 & $-1,013$ & 0,311 \\
\cline { 2 - 10 } & Mujeres & 60 & 35,04 & 1331,50 & 35,3684 & 7,18640 & & &
\end{tabular}

La ansiedad es un estado y es un rasgo, como estado las personas podemos aprender técnicas de autocontrol y también se puede desarrollar agentes externos de control; sin embargo la estabilización de los comportamientos de control presentan mayor dificultad que cuando se aprendieron como conductas aversivas, por ello los entrenamientos logran modificaciones pero no lo suficientemente como para recomendar técnicas por ello en el presente estudio se propuso verificar la medida en que se puede lograr estabilizar los autocontrol de la ansiedad, registrándose valores promedio de 35 puntos que corresponde a niveles de ansiedad dentro de lo normal (ver tabla 2)

Tabla 2.

Descriptivos de valores de ansiedad

\begin{tabular}{llcccccccc}
\hline Grupo & $\mathrm{N}$ & $\begin{array}{c}\text { Rango } \\
\text { promedi } \\
\text { o }\end{array}$ & $\begin{array}{c}\text { Suma } \\
\text { de } \\
\text { rangos }\end{array}$ & Media & $\begin{array}{c}\text { Desviac. } \\
\text { estándar }\end{array}$ & $\begin{array}{c}\text { Ude } \\
\text { Mann- } \\
\text { Whitne } \\
\text { y }\end{array}$ & Z & $\begin{array}{c}\text { Sig. } \\
\text { asintóti } \\
\text { ca } \\
\text { (bilater } \\
\text { al) }\end{array}$ \\
\hline $\begin{array}{l}\text { Ansiedad } \\
\text { Zung }\end{array}$ & Hombres & 79 & 37,49 & 1349,50 & 35,333 & 6,94879 & 683,500 & $-0,005$ & 0,996 \\
\cline { 2 - 10 }
\end{tabular}

Según los datos de la tabla 3, se encontró una correlación negativa significativa entre el nivel de ansiedad y los hábitos de estudio, lo que significa que el grupo de personas que reportaron mayores niveles de ansiedad presentaron a su vez menores niveles de hábitos de estudio (Tabla $3)$. 
Tabla 3.

Comparación de Hábitos de estudio y ansiedad

Hábitos de estudio
$-.170^{* *}$

Ansiedad

** Sig. al p. $<0.1$

\section{Discusión}

En la presente investigación se encontró que existe una correlación negativa significativa entre el nivel de ansiedad y los hábitos de estudio, estudios similares encontramos en Gil-Tapia y BotelloPríncipe (2018) encontrándose en su estudio correlacional sobre procrastinación académica y ansiedad en estudiantes de las carreras profesionales de Ciencias de la Salud, una asociación significativa entre los niveles de ansiedad y la dimensión de postergación de actividades $(\mathrm{p}=0.00)$, además se halló relación significativa entre la carrera profesional y la ansiedad, siendo los estudiantes de la carrera de Psicología los que están en los límites normales. Por otro lado, Cardona-Arias, Perez-Restrepo, Rivera-Ocampo, Gómez-Martínez y Reyes (2015) determinaron la prevalencia de ansiedad en estudiantes de una universidad privada de Colombia, identificando que la prevalencia de ansiedad presentó una distribución estadísticamente igual según sexo y grupo etario; además no presentó asociación con el ciclo de formación, el programa académico ni la ocupación. Dicha prevalencia solo presentó una asociación estadísticamente significativa con el estrato social, siendo menor en los estudiantes de clase media.

Son múltiples los estudios que han referido en población universitaria, que las mujeres tienden a puntuar más alto que los hombres en preguntas relacionadas con ansiedad. Incluso algunos han referido un riesgo mayor a 2,0 de padecer ansiedad en las mujeres frente a los hombres (Agudelo, Casadiegos \& Sánchez; 2008; Balanza, Morales y Guerrero, 2009).

Los resultados de la investigación permiten mencionar que la categoría pocos hábitos positivos de estudio son los que aparecen con mayor frecuencia en la muestra, por su parte Cruz y Quiñones (2011) encontraron en un estudio que buscaba determinar la relación entre los hábitos de estudio y el rendimiento académico en estudiantes de una facultad de ciencias de la salud en México, que el $85 \%$ de estudiantes poseían hábitos de estudio regulares y en el $15 \%$ son deficientes. Estos resultados llevan a la reflexión de diseñar estrategias para trabajar los hábitos de estudio desde el ingreso del estudiante a la universidad para mejora de su rendimiento académico.

El estudio encuentra que dentro del área ¿Cómo estudia Usted? existen menores índices de participación por parte de los estudiantes. Hernández, Rodríguez y Vargas (2012) encontraron en su estudio sobre hábitos de estudio y motivación para el aprendizaje en alumnos de tres carreras de ingeniería de México, que los alumnos tienen problemas por la organización y planeación del estudio, las técnicas de memorización, la comprensión de lectura, el estudiar en casa y la carencia de estrategias para incrementar la motivación y la autoestima. Por su parte, Chilca (2017) en su investigación sobre la relación entre la autoestima, los hábitos de estudio con el rendimiento académico de estudiantes universitarios de Lima encontraron que existe una relación significativa entre los hábitos de estudios y el rendimiento académico de los estudiantes en la población de estudio ( $\mathrm{R}=0.503$ ), además se determinó que cerca del $50 \%$ de los estudiantes tienen que modificar sus técnicas de estudio ya que son inadecuados.

Se hace urgente y necesario trabajar los hábitos de estudio desde el momento en que el estudiante andragogo inicia su vida universitaria, para ello, se deben diseñar estrategias plasmadas en un programa universitario que planteen objetivos y metas a corto, mediano y largo plazo y así evitar la deserción por los posibles resultados negativos que obtendrían desde su primer ciclo. El escaso tiempo del que dispondría el estudiante andragogo sumado a las nuevas responsabilidades 
académicas impedirían que se acojan a un programa universitario donde se den a conocer estrategias para mejorar los hábitos de estudio, por ello, se recomendaría que dicho programa sea parte del plan de estudios de todas las carreras.

\section{Referencias}

Agudelo, D., Casadiegos, C., \& Sánchez, D. (2008). Características de ansiedad y depresión en estudiantes universitarios. International Journal of Psychological Research, 1(1), 3439.

Alarcón-Diaz, H., Zapata, N. A., Díaz, M. A., Fernández, Y. O., Hernández, R. M., \& Rodríguez, A. (2019). Influencia de un programa para el desarrollo del aprendizaje experiencial en agricultores. Avances en Psicología, 27(1), 65-72. Recuperado de http://revistas.unife.edu.pe/index.php/avancesenpsicologia/article/view/1469

Alcalá, A. (1999). Es la andragogía una ciencia. Ponencia. Postgrado UNA Caracas, Venezuela. Recuperado de http://dip.una.edu.ve/mead/andragogia/paginas/alcala1997.pdf

Balanza, S., Morales, I., \& Guerrero, J. (2009). Prevalencia de ansiedad y depresión en una población de estudiantes universitarios: Factores académicos y socio-familiares asociados. Cínica y Salud, 20(2), 177-187.

Cardona-Arias, J. A., Perez-Restrepo, D., Rivera-Ocampo, S., Gómez-Martínez, J., \& Reyes, Á. (2015). Prevalencia de ansiedad en estudiantes universitarios. Diversitas: Perspectivas en Psicología, 11(1), 79-89.

Chilca, L. (2017). Autoestima, hábitos de estudio y rendimiento académico en estudiantes universitarios. Propósitos y Representaciones, 5(1), 71-127 doi: http://dx.doi.org/10.20511/pyr2017.v5n1.145

Cruz, F., \& Quiñones, A. (2011). Hábitos de estudio y rendimiento académico en enfermería, Poza Rica, Veracruz, México. Revista Electrónica Actualidades Investigativas en Educación, 11(3), 1-17.

Gil-Tapia, L., \& Botello-Príncipe, V. (2018). Procrastinación académica y ansiedad en estudiantes de Ciencias de la Salud de una Universidad de Lima Norte. CASUS. Revista de Investigación y Casos en Salud, 3(2), 89-96. doi: https://doi.org/10.35626/casus.2.2018.75

Hernández, C., Rodríguez, N., \& Vargas, A. (2012). Los hábitos de estudio y motivación para el aprendizaje de los alumnos en tres carreras de ingeniería en un tecnológico federal de la ciudad de México. Revista de la educación superior, 41(163), 67-87.

Paredes, M. A. (2018). Hábitos de estudio y motivación educativa en estudiantes de la universidad ESAN. Recuperado de http://www.repositorioacademico.usmp.edu.pe/bitstream/usmp/4126/1/paredes_bma.pdf

Salazar García, F. R. (2018). Ansiedad y Bienestar Psicológico en los adolescentes de la IE José Carlos Mariátegui, Trujillo. Universidad Católica de Trujillo Benedicto XVI. Recuperado de http://190.223.196.26/handle/123456789/313

Sandoval, J. (2019). Retos y desafíos en un ambiente Blended para el aprendizaje de las matemáticas de los primeros ciclos de estudiantes adultos. Universidad de Los Andes, Colombia. Recuperado de http://funes.uniandes.edu.co/14116/

Silva, F. D. J. C. (2018). Andragogía, andragogos y sus aportaciones. Voces de la educación, 3(6), 64-76.

Recuperado

de https://revista.vocesdelaeducacion.com.mx/index.php/voces/article/view/120/107.

Suárez Vera, L. A. (2018). Optimismo y ansiedad en estudiantes de una Universidad Estatal de Lima. Universidad Nacional Federico Villarreal, Lima. Recuperado de http://repositorio.unfv.edu.pe/bitstream/handle/UNFV/2651/SUAREZ\%20VERA\%20L UIGI\%20ALBERTO.pdf? sequence $=1 \&$ isAllowed $=\mathrm{y}$

Tahua, G., \& Cecilia, M. (2018). La educación de personas jóvenes y adultas en el mundo, en América Latina y en el Perú. Universidad de Educación Enrique Guzmán y Valle, Lima. Recuperado de http://repositorio.une.edu.pe/handle/UNE/3654

UNESCO. (1976). The belgrade charter. Connect UNESCO-UNEP Environ. Educ. Newsl, 1, pp. 1-2. 
Vicuña, P. (2014). Inventario de Hábitos de Estudio CASM-85-R-2014. Lima: GRAFI-K 